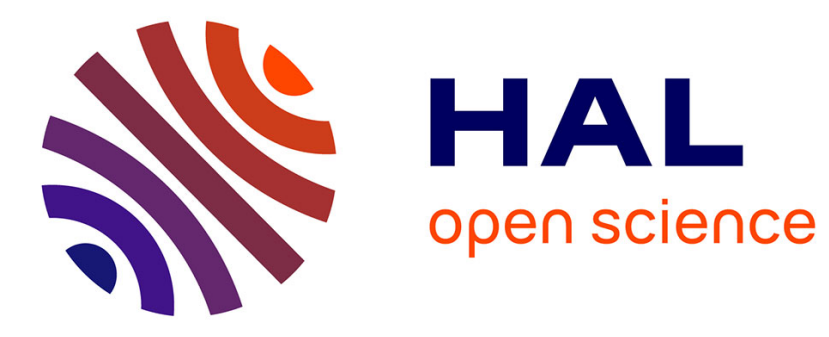

\title{
The win-first probability under interest force
}

Didier Rullière, Stéphane Loisel

\section{To cite this version:}

Didier Rullière, Stéphane Loisel. The win-first probability under interest force. Insurance: Mathematics and Economics, 2005, 37 (3), pp.421-442. 10.1016/j.insmatheco.2005.06.004 . hal-00165791

\section{HAL Id: hal-00165791 \\ https://hal.science/hal-00165791}

Submitted on 27 Jul 2007

HAL is a multi-disciplinary open access archive for the deposit and dissemination of scientific research documents, whether they are published or not. The documents may come from teaching and research institutions in France or abroad, or from public or private research centers.
L'archive ouverte pluridisciplinaire HAL, est destinée au dépôt et à la diffusion de documents scientifiques de niveau recherche, publiés ou non, émanant des établissements d'enseignement et de recherche français ou étrangers, des laboratoires publics ou privés. 


\title{
Institut de Science Financière et d'Assurances
}

\author{
Les Cahiers de Recherche de I'ISFA
}

THE WIN-FIRST PROBABILITY UNDER INTEREST FORCE

Didier Rullière

Stéphane Loisel

Cahier de recherche WP 2029 (2005)

Université Claude Bernard Lyon 1

Retrouvez la liste complète des cahiers de recherche de l'ISFA à l'adresse :

http://isfaserveur.univ-lyon1.fr/cahiers-recherche-isfa/ 


\title{
The win-first probability under interest force
}

\author{
Didier Rullière, Stéphane Loisel* \\ Ecole ISFA - 50, avenue Tony Garnier - 69366 Lyon Cedex 07, France
}

\begin{abstract}
In a classical risk model under constant interest force, we study the probability that the surplus of an insurance company reaches an upper barrier before a lower barrier. We define this probability as win-first probability. Borrowing ideas from life-insurance theory, hazard rates of the maximum of the surplus before ruin, regarded as a remaining future lifetime random variable, are studied, and provide an original derivation of the win-first probability. We propose an algorithm to efficiently compute this risk-return indicator and its derivatives in the general case, as well as bounds of these quantities. The efficiency of the proposed algorithm is compared with adaptations of other existing methods, and its interest is illustrated by the computation of the expected amount of dividends paid until ruin in a risk model with a dividend barrier strategy.
\end{abstract}

Key words: Ruin probability, hazard rate, upper absorbing barrier, constant interest force, risk-return indicator, win-first probability.

JEL Classification codes: G22, C60.

* Corresponding author. Tel. +3343728 74 38, fax +3343728 7632

Email addresses: Didier.Rulliere@univ-lyon1.fr (Didier Rullière), Stephane.Loisel@univ-lyon1.fr (Stéphane Loisel). 


\section{Introduction}

In this paper, we propose a way to compute the probability that a risk process reaches an upper barrier (representing a goal or a threshold for a dividend policy) before crossing a lower barrier (representing the ruin of the company, or a threshold for insolvency penalties). We define this probability as win-first probability.

We consider the compound Poisson risk model with a constant instantaneous interest force $\delta$. The surplus of an insurance company at time $t$ is modeled by the process $R_{t}$, where $R_{0}=u$ and $R_{t}$ satisfies the stochastic differential equation:

$$
d R_{t}=c d t-d S_{t}+\delta R_{t} d t
$$

Here, $u$ is the initial surplus, $c$ the premium income rate, and the cumulated claims process $S_{t}$ is a compound Poisson process given by the Poisson parameter $\lambda$ and the distribution function $F_{W}$ of the individual claim amount $W$, with mean $m$. Assume that $c>\lambda m$. Denote by $T_{u}$ and $T_{u}^{v}$ the respective times to lower or upper barrier, with initial surplus $u$,

$$
T_{u}=\inf \left\{t, R_{t}<0\right\} \quad \text { and } \quad T_{u}^{v}=\inf \left\{t, R_{t} \geq u+v\right\},
$$

with $T_{u}=+\infty$ if $\forall t \geq 0, R_{t} \geq 0$ and $T_{u}^{v}=+\infty$ if $\forall t \geq 0, R_{t}<u+v$. The non-ruin probability within finite time $t$ is

$$
\varphi_{\delta}(u, t)=\mathbb{P}\left(T_{u}>t\right)
$$

and the eventual non-ruin probability and ruin probability are respectively

$$
\varphi_{\delta}(u)=\mathbb{P}\left(T_{u}=+\infty\right) \quad \text { and } \quad \psi_{\delta}(u)=1-\varphi_{\delta}(u) .
$$

As $c>\lambda m, c t-S_{t} \stackrel{a . s .}{\rightarrow}+\infty$ as $t \rightarrow \infty$. If $\delta=0$ (no interest force), for any $(u, v) \in \mathbb{R}^{2}, T_{u}^{v}$ is an almost surely finite stopping time and one can determine whether or not $T_{u}>T_{u}^{v}$. However, if $\delta>0$,

$$
\mathbb{P}\left(R_{t} \rightarrow+\infty \text { as } t \rightarrow+\infty\right) \neq 1,
$$

because there exists a threshold $y<0$ such that, if for some $t>0, R_{t}<y$, then surely $\forall s>t, R_{s}<0$. This corresponds to the definition of ruin under interest force of Gerber (1979). This phenomenon causes many generalizations of the classical risk model to fail.

Nevertheless, if for all $t \geq 0, R_{t} \geq 0$, then $R_{t} \stackrel{\text { a.s. }}{\rightarrow}+\infty$ as $t \rightarrow \infty$. This will be very important to compute the win-first and the lose-first probabilities with constant interest force, respectively defined as

$$
\begin{aligned}
& \mathrm{WF}(u, v)=\mathbb{P}\left(T_{u}^{v}<T_{u}\right), \\
& \mathrm{LF}(u, v)=1-\mathrm{WF}(u, v) .
\end{aligned}
$$


These probabilities may provide risk and profit indicators with the same unit: subjectivity is reduced to the choice of the lower bound $u$, which represents the event "lose", and the upper bound $v$, which represents the event "win". Without upper barrier, one drawback of the probability of ruin is that its minimization often prescribes the cession of the whole activity by the insurer to the reinsurer. Besides, it does not give any information about the possible profit, even for very small ruin probabilities. It is interesting to combine it with a return indicator, and one of the simplest compromises is to consider the probability $\operatorname{WF}(u, v)$ to reach a level $u+v$ from initial surplus $u$ before being ruined. It has the advantage not to require constrained optimization techniques.

Risk and return indicators can be built from the win-first probability, such as the initial surplus required to avoid a failure, $u_{\varepsilon}(v)=\inf \{u, 1-\mathrm{WF}(u, v) \leq \varepsilon\}$, the objective level $v$ and confidence level $\varepsilon$ being given, or the maximal objective level that is reasonably achievable $v_{\varepsilon}(u)=\sup \{v, \operatorname{WF}(u, v) \geq 1-\varepsilon\}, u$ and $\varepsilon$ being given. The two barriers thus help to define synthetic risk-return indicators having the same unit, like $\left(u_{\varepsilon}(v), v\right)$ and $\left(u, v_{\varepsilon}(u)\right)$, useful to compare reinsurance or investment strategies. Other quantities involving win-first probabilities can be considered, such as $\mathbb{E}\left(\left(T_{u}-T_{u}^{v}\right)_{+}\right), \mathbb{E}\left(\left(T_{u}^{v}-T_{u}\right)_{+}\right) \ldots$

Double barrier problems have been studied in the compound Poisson model without interest force by Segerdahl (1970), Dickson and Gray (1984a,b), Wang and Politis (2002). We first give properties of win-first probabilities in subsection 2.1, including a differential equation and a direct adaptation of a result of Segerdahl (1942). We thus obtain the win-first probability as a quotient of two non-ruin probabilities. A first way to tackle the problem of numerically compute win-first probabilities would be to use existing methods (Brekelmans and De Waegenaere (2001), Sundt and Teugels (1995, 1997), De Vylder (1999)) of computing ruin probabilities for some particular claim amount distributions, or for small $\delta$, and to take the quotient. For exponentially distributed claim amounts, the probability of ruin under constant interest force is well-known (see Segerdahl (1942), or Sundt and Teugels (1995)). For general claim size distribution, bounds and Lundberg coefficients have been derived by Sundt and Teugels $(1995,1997)$, and several others.

Sundt and Teugels (1997) obtain bounds for the adjustment function. Konstantinides et al. (2002) obtain an asymptotical two-sided bound for heavytailed claim size distribution from generalizing results of the classical case $\delta=0$ to the general case. It is possible to use these bounds to get a two-sided bound for the win-first probability with interest force with heavy-tailed claim size distribution. However, we do not need in our problem to compute ruin probabilities, and we shall introduce an original method which is adapted to the present framework and more suitable in the general case and for general interest force $\delta$ than the method consisting in computing the two corresponding ruin probabilities. 
The formulation of the problem, and the quotient of survival probabilities suggest the possibility to study a ruin-related survival function of some defective random variable $\theta$, inspired from life-insurance theory. We study in subsection 2.2 its hazard rate function and propose an algorithm to compute the win-first probabilities and its derivatives, and a bound of the numerical error. A particular property of the hazard rates of $\theta$ (see theorem 5) is the key argument which makes the method so efficient. The algorithm and reasons for someone to want to use it are detailed in section 3. In section 4, numerical examples are given to demonstrate the accuracy of the algorithm and applications are proposed. In particular, computing expressions like $\mathbb{E}[\mathrm{WF}(u-W, W)]$ which involve win-first probabilities are of real interest in models with dividends. For example, Frostig (2004) and Gerber and Shiu (1998), considered risk models with a dividend barrier, and computed the expected amount of dividends until time $t$ and until ruin, or optimal dividend strategies. These quantities are expressed in subsection 4.1 in terms of win-first probabilities, which correspond in this framework to the probability that the dividends are positive. We compare our method with the one using Sundt and Teugels (1995) in subsection 4.3 .

\section{Win-first probability}

In this section, we first adapt classical results of ruin theory to our framework. There is no essentially new idea in subsection 2.1. This is the reason why we only state the results we shall need later. The proofs are similar as in the case $\delta=0$. We introduce in subsection 2.2 the new method we propose to compute the win-first probabilities in the general case.

\subsection{Adaptation of classical results and methods of ruin theory}

Note that $\operatorname{WF}(u, v)$ is nondecreasing with respect to $u$, nonincreasing with respect to $v$, and that

$$
\begin{array}{ll}
\mathrm{WF}(u, v)=0 & \text { for all } u<0, \quad \text { and } \\
\mathrm{WF}(u, v)=1 & \text { for all } u \geq 0, v \leq 0 .
\end{array}
$$

Remark 1 In the special case $\delta=0, R_{t}=u+c t-S_{t}$ corresponds to the classical risk process, and $R_{t}-R_{0}$ does not depend on $R_{0}=u$. In this case, $u$ is not necessarily the initial reserve, and $\mathrm{WF}(u, v)$ corresponds to the probability that the surplus process $R_{t}$ reaches $R_{0}+v$ before reaching the barrier $R_{0}-u$, and does not depend on $R_{0}$. 
Theorem 1 For $v \geq 0, w \geq 0$,

$$
\mathrm{WF}(u, v+w)=\mathrm{WF}(u, v) \cdot \mathrm{WF}(u+v, w) .
$$

Proof: For $u \geq 0, v>0, w>0$, from stationarity and Markov property of $R_{t}$, earning $v+w$ before losing $u$ may be decomposed into: earning $v$ before losing $u$ and then earning $w$ before losing $u+v$. If $v=0$ or $w=0$ equality is obvious. For $u<0$, both terms are equal to 0 .

Theorem 2 For $u \geq 0, v>0$,

$$
\begin{aligned}
& \frac{\partial}{\partial u} \operatorname{WF}(u, v)-\frac{\partial}{\partial v} \operatorname{WF}(u, v)=\frac{\lambda}{c+\delta u}(\mathrm{WF}(u, v)-\mathbb{E}[\mathrm{WF}(u-W, v+W))] \\
& \frac{\partial}{\partial u} \operatorname{WF}(u, v)-\frac{\partial}{\partial v} \operatorname{WF}(u, v)=\frac{\lambda}{c+\delta u} \operatorname{WF}(u, v) \cdot(1-\mathbb{E}[\mathrm{WF}(u-W, W)]) .
\end{aligned}
$$

Proof: From Poisson process properties, we get

$$
\begin{gathered}
\mathrm{WF}(u, v)=(1-\lambda \Delta t) \cdot \mathrm{WF}\left(u e^{\delta \Delta t}+\frac{c}{\delta}\left(e^{\delta \Delta t}-1\right), v-\left(u+\frac{c}{\delta}\right)\left(e^{\delta \Delta t}-1\right)\right) \\
+\lambda \Delta t \cdot \mathbb{E}\left[\mathrm{WF}\left(u e^{\delta \Delta t}+\frac{c}{\delta}\left(e^{\delta \Delta t}-1\right)-W, v-\left(u+\frac{c}{\delta}\right)\left(e^{\delta \Delta t}-1\right)+W\right)\right]+o(\Delta t) .
\end{gathered}
$$

This heuristic argument shows that equation (2) may be derived with classical ruin theory tools. For $u=0$, we take the convention that $\frac{\partial}{\partial u} \mathrm{WF}(u, v)$ is the right derivative of $\operatorname{WF}(u, v)$. Note that in this case, the last term of equation (2) disappears. Starting from (2), a direct application of (1) leads to

$$
\mathrm{WF}(u-W, v+W)=\mathrm{WF}(u-W, W) \cdot \mathrm{WF}(u, v),
$$

which provides the second equation.

Inequalities between win-first probabilities and some finite-time ruin probabilities may be derived.

Proposition 1 For any $u \geq 0, v \geq 0$, we have

$$
\varphi_{\delta}(u) \leq \mathrm{WF}(u, v) \leq \varphi_{\delta}\left(u, \tau_{\delta}(u, v)\right)
$$

where $\quad \tau_{\delta}(u, v)=\frac{1}{\delta} \ln \left(1+\frac{v}{u+c / \delta}\right) \quad$ if $\quad \delta>0, \quad$ and $\quad \tau_{0}(u, v)=v / c$.

Proof: For $u \geq 0, v \geq 0$, if $T_{u}=+\infty$ then the insurer earns almost surely $v$ before losing $u$, because $R_{t} \stackrel{a . s}{\rightarrow}+\infty$ as $t \rightarrow \infty$. It follows $\operatorname{WF}(u, v) \geq$ $\mathbb{P}\left(T_{u}=+\infty\right)=\varphi_{\delta}(u)$. Now, if the insurer earns $v$ before losing $u$, time needed 
to earn $v$ is necessarily greater than the solution $\tau_{\delta}(u, v)$ of equation in $t$ :

$$
u e^{\delta t}+\frac{c}{\delta}\left(e^{\delta t}-1\right)=u+v
$$

and $T_{u}>\tau_{\delta}(u, v)$. So, $\operatorname{WF}(u, v) \leq \mathbb{P}\left[T_{u}>\tau_{\delta}(u, v)\right]$.

Finally, considering $\lim _{v \rightarrow \infty} \mathrm{WF}(u, v)$, enables us to express $\mathrm{WF}(u, v)$ as a quotient of survival probabilities.

Theorem 3 For $u \geq 0, v \geq 0$,

$$
\mathrm{WF}(u, v)=\frac{\varphi_{\delta}(u)}{\varphi_{\delta}(u+v)} .
$$

In the special case $\delta=0$, this result has been recently developed by Wang and Politis (2002), and had also been treated previously by Dickson and Gray (1984b) and Segerdahl (1970). The idea is here exactly the same, and we omit the proof of the extension, which is rather direct.

From equation (5), it is possible to derive an exact formula for $\operatorname{WF}(u, v)$ in the case of exponentially distributed claim amounts (see Segerdahl (1942), or Sundt and Teugels (1995)), and asymptotical equivalents and bounds for general claim size distribution, as mentioned in the introduction.

\subsection{Hazard rates of $\theta$ and applications}

In this section we present an interesting interpretation of $\mathrm{WF}(u, v)$. Let us change our notation for an instant and write

$$
{ }_{v} p_{u}=\mathrm{WF}(u, v) .
$$

Property (1) can be written

$$
{ }_{v+w} p_{u}={ }_{v} p_{u} \cdot{ }_{w} p_{u+v},
$$

and corresponds to a simple classical formula, expressed in International Actuarial Notation (see Actuarial Mathematics), stating that for a positive future lifetime $\theta$,

$\mathbb{P}(\theta \geq u+v+w \mid \theta \geq u)=\mathbb{P}(\theta \geq u+v \mid \theta \geq u) \cdot \mathbb{P}(\theta \geq u+v+w \mid \theta \geq u+v)$

This formula, based on elementary conditioning, illustrates the fact that someone aged $u$ survives $v+w$ years, if he first survives $v$ years, and, being then aged $u+v$, survives $w$ more years. So, it seems logical to look for a nonnegative random variable $\theta$ such that $\mathrm{WF}(u, v)=\mathbb{P}(\theta \geq u+v \mid \theta \geq u)$. Let $\theta$ be the 


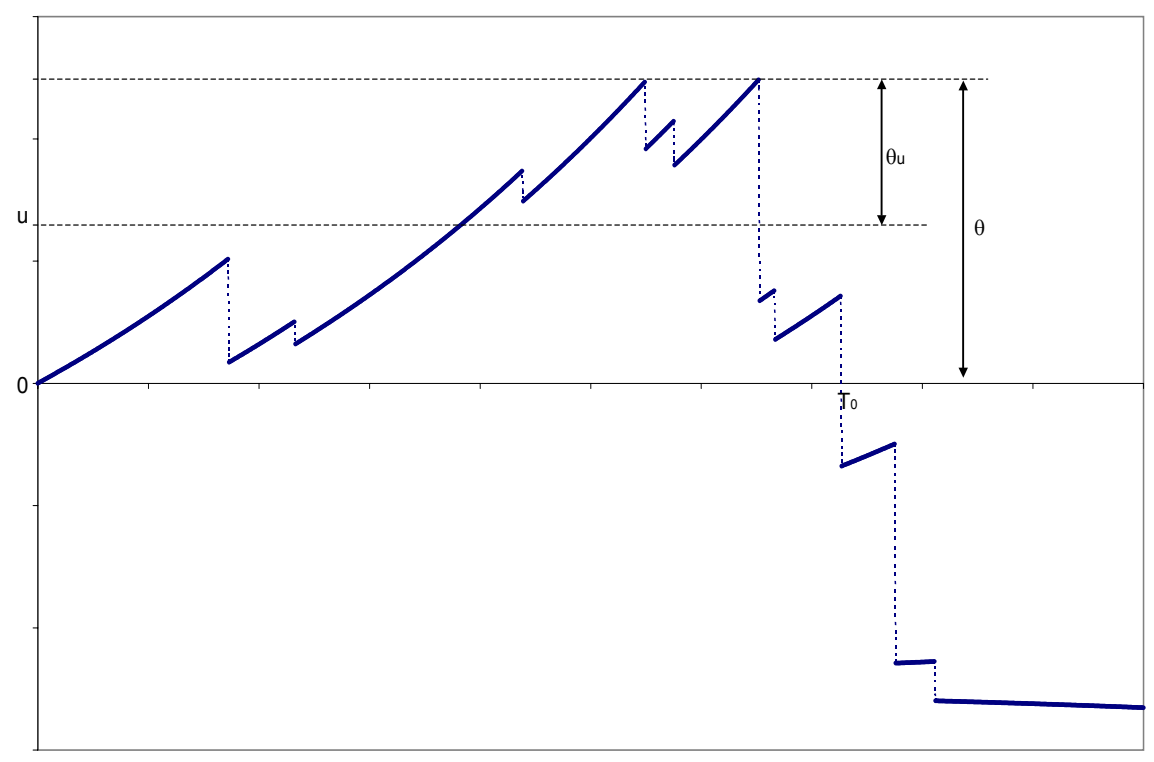

Figure 1. Sample path of $R_{t}$, with $\theta$ and $\theta_{u}=(\theta-u)_{+}(\delta=20 \%)$.

positive, defective random variable

$$
\theta=\sup \left\{R_{t}, t \leq T_{0} \mid R_{0}=0\right\}
$$

Define the survival function of $\theta$ by $S(x)=\mathbb{P}(\theta \geq x), x \in \mathbb{R}^{+}$, and its hazard rate by

$$
\mu_{x}=-\frac{S^{\prime}(x)}{S(x)}=-\frac{\partial}{\partial x} \ln (S(x))
$$

Theorem 4 For $u \geq 0, v \geq 0$, the win-first probability can be written as

$$
\begin{aligned}
\mathrm{WF}(u, v) & =\mathbb{P}(\theta \geq u+v \mid \theta \geq u)=S(u+v) / S(u), \\
\text { with } S(x) & =\mathbb{P}(\theta \geq x)=\varphi_{\delta}(0) / \varphi_{\delta}(x), \quad x \geq 0 .
\end{aligned}
$$

Proof: Let us first consider the case $u=0, v \geq 0$. If $T_{0}=+\infty, R_{t} \stackrel{\text { a.s. }}{\rightarrow}+\infty$ as $t \rightarrow \infty$, and upper barrier $v$ is reached after an almost surely finite time $T_{u}^{v}<T_{0}$. In this case, given that $T_{0}=+\infty, W F(0, v)=1=\mathbb{P}(\theta \geq v)$. If $T_{0}<+\infty$, upper barrier $v$ is reached if and only if $\theta \geq v$, and $\operatorname{WF}(0, v)=$ $\mathbb{P}(\theta \geq v)$. In every case $\operatorname{WF}(0, v)=\mathbb{P}(\theta \geq v), v \geq 0$. Consider now $u \geq 0$, $v \geq 0$. We have seen that $T_{0}=+\infty$ implies $\theta \geq u$. So $\mathbb{P}(\theta \geq u) \geq \varphi_{\delta}(0)>$ 0 . Starting from property (1), we have $\operatorname{WF}(u, v)=\mathrm{WF}(0, u+v) / \mathrm{WF}(0, u)=$ $\mathbb{P}(\theta \geq u+v) / \mathbb{P}(\theta \geq u)$. And the result is obvious since $v \geq 0$.

For $u \geq 0, v \geq 0$, note that

$$
\mu_{u+v}=-\frac{\partial}{\partial v} \ln \mathrm{WF}(u, v)
$$


This rate is finite and only depends on the sum $u+v$. In the case of integervalued claim amounts, we will see that $\mu_{u}$ is continuous and derivable at each $u \in \mathbb{R}^{+} \backslash \mathbb{N}$. For $u \in \mathbb{N}, \mu_{u}$ will be only right-continuous and rightdifferentiable, so that we will take the convention that each derivative of $\mu$ is its right derivative. We will take the same convention for derivatives in $u$ of $\mathrm{WF}(u, v)$. Given that $\theta \geq u$, the conditional density of $\theta$ is

$$
f_{\theta_{u}}(x)=\frac{\partial}{\partial x} \mathbb{P}(\theta<u+x \mid \theta \geq u)=W \mathrm{~F}(u, v) \cdot \mu_{u+v}
$$

Hence, for example, $\operatorname{LF}(u, v)=\mathbb{P}(\theta<u+v \mid \theta \geq u)=\int_{0}^{v} \mathrm{WF}(u, s) \mu_{u+s} d s$.

In the sequel, since we will use common actuarial tools, we will most often preferably write probabilities with standard actuarial notations, using ${ }_{t} p_{x}$ instead of $\mathrm{WF}(x, t)$, and will also write:

$$
\mu_{u}^{(i)}=\frac{\partial^{i}}{\partial u^{i}} \mu_{u}, \quad S_{u}^{(i)}=\frac{\partial^{i}}{\partial u^{i}} S(u), \quad{ }_{t} p_{x}^{(i)}=\frac{\partial^{i}}{\partial x^{i}}{ }_{t} p_{x}, \quad{ }_{w} p_{u-w}^{(i)}=\frac{\partial^{i}}{\partial u^{i}}{ } p_{u-w}
$$

Note that, due to these definitions, we do not have an equality between $S_{u}^{(i)}$ and ${ }_{w} p_{u-w}^{(i)}$ when $w=u$.

Let us denote by $C_{n}^{k}$ the binomial coefficient for integers $k$ and $n, 0 \leq k \leq n$.

Proposition 2 For $u, v \geq 0$, we have

$$
\begin{aligned}
\mathrm{WF}(u, v) & =\exp -\int_{u}^{u+v} \mu_{s} d s, \\
{ }_{t} p_{x}^{(1)} & ={ }_{t} p_{x}\left(\mu_{x}-\mu_{x+t}\right), \\
{ }_{t} p_{x}^{(k+1)} & =\sum_{i=0}^{k} C_{k t}^{i} p_{x}^{(i)}\left(\mu_{x}^{(k-i)}-\mu_{x+t}^{(k-i)}\right), \quad k \geq 0 .
\end{aligned}
$$

Proof: (7) holds directly from theorem 4. Differentiations are straightforward.

Proposition 3 A general link between unconditional survival function and hazard rate is given for $x \geq 0, k \geq 0$ by

$$
\begin{aligned}
S^{(1)}(x) & =-\mu_{x} S(x), \\
S^{(k+1)}(x) & =-\sum_{i=0}^{k} C_{k}^{i} \mu_{x}^{(i)} S^{(k-i)}(x) .
\end{aligned}
$$

Theorem 5 The hazard rate of $\theta$ and its right derivatives are as follows: 


$$
\begin{aligned}
\mu_{u} & =\alpha_{u}\left(1-\mathbb{E}\left({ }_{W} p_{u-W}\right)\right), \\
\mu_{u}^{(k)} & =\alpha_{u}^{(k)}-\sum_{j=0}^{k} C_{k}^{j} \alpha_{u}^{(k-j)} \mathbb{E}\left({ }_{W} p_{u-W}^{(j)}\right), \quad \text { for } u \geq 0, k \geq 0 . \\
\text { with } \alpha_{u}^{(k)} & =k ! \lambda(-\delta)^{k}(c+\delta u)^{-(k+1)}, \alpha_{u}=\alpha_{u}^{(0)} .
\end{aligned}
$$

Proof: direct from (3) and from (7).

Note that first equation in previous relations could also be written:

$$
\begin{aligned}
\mu_{u} & =\frac{\lambda}{c+\delta u}\left(1-\mathbb{E}\left[\mathbb{1}_{W \leq u} \exp -\int_{u-W}^{u} \mu_{s} d s\right]\right), \\
\mu_{u} & =\frac{\lambda}{c+\delta u}\left(\left(1-F_{W}(u)\right)+\mathbb{E}\left[\mathbb{1}_{W \leq u} \cdot \operatorname{LF}(u-W, W)\right]\right) .
\end{aligned}
$$

In particular, suppose $W$ is a continuous random variable. It is clear that $\mathrm{WF}(u-W, W)=0$ if $W>u$. It follows from (5) that $\mu_{0}=\frac{\lambda}{c}$ and that $\forall u \geq 0$, $0 \leq \mu_{u} \leq \mu_{0}$. Since $\mathrm{WF}(0, v)=\exp -\int_{0}^{v} \mu_{s} d s>0$ for each $v>0, \mu_{+\infty}=$ $\lim _{u \rightarrow+\infty} \mu_{u}=0$. Furthermore, differentiation of $\mu_{u}$ follows immediately from (7) and (5).

Hence, when $W$ is a continuous random variable, the hazard rate $\mu_{u}$ is a continuous, decreasing function of $u$, such that

$$
\mu_{0}=\frac{\lambda}{c}, \quad \lim _{u \rightarrow+\infty} \mu_{u}=0, \quad \mu_{0}^{\prime}=-\frac{\lambda \delta}{c^{2}} \quad \text { and } \quad \lim _{u \rightarrow+\infty} \mu_{u}^{\prime}=0 .
$$

Remark 2 For $\delta=0$, differentiation of $\mathrm{WF}(u, v)$ makes sense, and computing $\mu_{u}, u>0$ in terms of $\varphi_{0}(u)$ leads to

$$
\mu_{u}=\varphi_{0}^{\prime}(u) / \varphi_{0}(u) .
$$

We also check that, in the special case $\delta=0$, formula (5) is a version of the classical risk theory formula

$$
\varphi_{0}^{\prime}(u)=\frac{\lambda}{c} \varphi_{0}(u)-\frac{\lambda}{c} \mathbb{E}\left[\varphi_{0}(u-W)\right] .
$$

\section{Algorithm}

The recursive determination of hazard rate $\mu_{u}$ and its derivatives, for successive values of $u$, gives a set of values of $S(u)$ and its derivatives up to a given order. Despite the purpose is here to find values of win-first probabilities, this will eventually give results on $\psi_{\delta}(u)$ when $\psi_{\delta}(0)$ is known. 
The proposed iterative algorithm allows to re-use previous computed quantities, and then reduce the complexity of the determination of the whole function $S$. It gives many derivatives of $\mu_{u}$ and $S(u)$, and all numerical errors will be bounded in a further section. No assumption is made neither on claim amounts nor on the interest force $\delta$, making the context different from studies using small $\delta$ (see Sundt and Teugels (1995) and section 4.3), and from the one using particular distributions for claim amounts (see Konstantinides et al. (2002) and Brekelmans and De Waegenaere (2001))

\subsection{Approximations}

In the sequel, $W$ is assumed to be a random variable taking values in the set $\mathbb{N}^{*}$ of positive integers. Define $\pi_{i}=\mathbb{P}(W=i), i \in \mathbb{N}$.

This hypothesis is not so stringent: in practice, we may approach any continuous random variable by a discrete one, and the discretization step may be chosen as small as necessary. Instead of taking this step smaller than 1, we choose this step equal to 1 and change the monetary unit.

The restriction $\pi_{0}=0$ can be easily eliminated: if $\pi_{0}>0$, one may replace $\pi_{0}, \pi_{1}, \pi_{2} \ldots$ with $0, \pi_{1} /\left(1-\pi_{0}\right), \pi_{2} /\left(1-\pi_{0}\right) \ldots$ and $\lambda$ with $\lambda\left(1-\pi_{0}\right)$ (see De Vylder, 1999).

The main assumption we shall use for approximations is :

Assumption $H_{r}^{\varepsilon}: \mu$ is locally polynomial of order $r$ on intervals $[k \varepsilon, k \varepsilon+\varepsilon[$, $k \in \mathbb{N}$.

We shall see further that even a choice like $r=2$ and $\varepsilon=0.5$ gives numerically quite good results (see section 4.2), and the precision of the algorithm increases rapidly for a better choice of these two parameters. In section 3.3, we will derive bounds for each approximated quantity in the algorithm, in order to ensure the numerical validity of this assumption. Under $H_{r}^{\varepsilon}$, we get from the $r$ first derivatives of $\mu$ :

$$
\begin{aligned}
\forall s \in[0, \varepsilon[, \mu(x+s) & =\sum_{i=0}^{r} \frac{\mu^{(i)}(x)}{i !} s^{i} \\
\text { For } x \in \varepsilon \mathbb{N}, S(x+\varepsilon) & =S(x) \exp \left(-\int_{0}^{\varepsilon} \sum_{i=0}^{r} \frac{\mu^{(i)}(x)}{i !} s^{i} d s\right), \\
S(x+\varepsilon) & =S(x) \exp \left(-\sum_{i=0}^{r} \frac{\mu^{(i)}(x)}{(i+1) !} \varepsilon^{i+1}\right) .
\end{aligned}
$$

We can also derive $S(x+\varepsilon)$ from derivatives of $S(x)$, but we choose to use the 
single hypothesis $H_{r}^{\varepsilon}$. In life insurance, the hypothesis of constant hazard rate is often considered for survival lifetimes, and corresponds here to the order $r=0$. In practice, it is possible to get higher order derivatives of $\mu_{u}$ since computation of $\mu_{u}^{(r+1)}$ may be replaced with an approximation like $\mu_{u}^{\text {left }(r)}=$ $\left(\mu_{u}^{(r)}-\mu_{u-\varepsilon}^{(r)}\right) / \varepsilon$. However, one should keep in mind that, if $W$ takes values in $\mathbb{N}$, each $x \in \mathbb{N}$ is a point of discontinuity for function $\mu$ (see figure 4). So, using this approximation will give good results, except for $u \in \mathbb{N}$. Nevertheless, since numerical results are fine enough, and since the parameter $r$ could be chosen, we did not use this approximation.

Proposition 4 (approximation algorithm) Under hypothesis $H_{r}^{\varepsilon}$, the following algorithm computes recursively the values of $S(u), \mu_{u}, \mathbb{E}\left[{ }_{W} p_{u-W}\right]$ and all their derivatives up to a given order $r$. With $S(0)=1$, and for $u \in \varepsilon \mathbb{N}$, $u \leq u_{\max }, k \in \mathbb{N}, k \leq r$,

$$
\begin{aligned}
{ }_{w} p_{u-w}^{(k)} & =\mathbf{1}_{\{k=0\}} \frac{S(u)}{S(u-w)}+\mathbf{1}_{\{k \geq 1\}} \sum_{i=0}^{k-1} C_{k-1 w}^{i} p_{u-w}^{(i)}\left(\mu_{u-w}^{(k-1-i)}-\mu_{u}^{(k-1-i)}\right), w=1 . .[u], \\
\mu_{u}^{(k)} & =\alpha_{u}^{(k)}-\sum_{j=0}^{k} C_{k}^{j} \alpha_{u}^{(k-j)} \mathbb{E}\left(W p_{u-W}^{(j)}\right), \\
S(u+\varepsilon) & =S(u) \exp \left(-\sum_{i=0}^{r} \frac{\mu^{(i)}(u)}{(i+1) !} \varepsilon^{i+1}\right) .
\end{aligned}
$$

From the second equation, quantities $\mu_{0}^{(0)}=\alpha_{0}^{(0)}$ and $\mu_{0}^{(k)}$ are given by the recursion, as the $\mathbb{E}\left({ }_{W} p_{u-W}^{(j)}\right), j \leq k$ are derived from previously computed quantities ${ }_{w} p_{u-w}^{(j)}$.

Note that $\alpha_{u}^{(k)}=k ! \lambda(-\delta)^{k}(c+\delta u)^{-(k+1)}$, and that for $w>u,{ }_{w} p_{u-w}^{(j)}=0$. The previous algorithm gives derivatives of $\mu$ from order 0 to $r$. It also gives for each $u \leq u_{\max }, u \in \varepsilon \mathbb{N}, S(u)$ and eventually $\psi_{\delta}(u)=1-\varphi_{\delta}(0) / S(u)$. To obtain derivatives of $\mathrm{WF}$ and $S$, we can use (8) for $\frac{\partial^{k}}{\partial u^{k}} \mathrm{WF}(u, v)$ and following relations:

$$
\begin{aligned}
\frac{\partial^{k}}{\partial v^{k}} \mathrm{WF}(u, v) & =S^{(k)}(u+v) / S(u), \\
S^{(k+1)}(x) & =-\sum_{i=0}^{k} C_{k}^{i} \mu_{x}^{(i)} S^{(k-i)}(x), k \geq 0 .
\end{aligned}
$$

Let $i$ be a positive integer. We have seen that $S(i)=\varphi_{\delta}(0) / \varphi_{\delta}(i)$. In the special case $\delta=0$, it is known that $\varphi_{0}(i)$ can be exactly computable by classical formulae (see Picard and Lefèvre (1997) and De Vylder (1999)): 


$$
\begin{aligned}
\varphi_{0}(i) & =\left(1-\frac{\lambda m}{c}\right) \sum_{j=0}^{i} h_{j}(j-i), \\
\text { with } h_{j}(\tau) & =\frac{\lambda \tau}{c j} \sum_{k=1}^{j} k \pi_{k} h_{j-k}(\tau) \text { and } h_{j}(0)=e^{-\frac{\lambda \tau}{c}} .
\end{aligned}
$$

This formula has the advantage to give exact values if $W$ is integer-valued. Let us compare the number of loops involved in the computation of $S(i), i=1 \ldots x$ by algorithm (4) and the number of loops involved in the computation of $\varphi_{0}(i), i=1 \ldots x$ by the Picard and Lefèvre (1997) algorithm. Computing $S(i), i=1 \ldots x$ implies $r^{2} / 2$ loops for $i=1 . . x / \varepsilon, j=1 \ldots i \varepsilon$, so that complexity of algorithm 4 is quite proportional to $r^{2} x^{2} / \varepsilon$. Computing $\varphi_{\delta}(i), i=1 \ldots x$ requires loops for $i=1 \ldots x, j=0 \ldots i, k=1 \ldots j$, so that complexity of PicardLefèvre formula is quite proportional to $x^{3}$. To approximate a continuous distribution $W$ by $W_{d}$ taking values in $d \mathbb{N}$, time needed by algorithm (4) is proportional to $r^{2} x^{2} /\left(d^{2} \varepsilon\right)$ against $x^{3} / d^{3}$ for the Picard and Lefèvre (1997) formula. Noting that hypothesis $H_{1}^{\varepsilon}$ means that $\mu$ is linear on intervals of length $d \varepsilon$, one can use $\varepsilon=1$ and $r=1$ if $d$ is small. As both formulae lead to an approximation of values obtained for a continuous $W$, the algorithm may be of a practical interest even in the case $\delta=0$. Moreover, we will see in section 3.4 that the complexity of the algorithm can be reduced in this case.

\subsection{Convergence for parameters $r$ and $n$}

The highest order of derivatives that are computed by the algorithm is $r$, and $n=1 / \varepsilon$ is an integer that represents the number of sub-periods in one unit of time. The hypothesis in approximation algorithm is that, on each sub-period, $\mu_{u}$ is locally polynomial of order $r$. The precision of the algorithm, at one step, is given by $\eta$, which represents the number of decimal digits that one aims at obtaining. More precisely, $10^{-\eta}$ represents the error in the approximation of $\mu_{u+\varepsilon}$ by the Taylor expansion of order $r$.

To improve the local precision of the algorithm, we can increase either $n$ or $r$; this may have different effects on the complexity of the algorithm. We only give here informal considerations for the choice of the couple $(n, r)$ to minimize the complexity of the algorithm. It would be possible to get more rigorous results for that choice of parameters, but they are omitted here in the interest of conciseness.

Note first that the remaining part in the Taylor expansion behaves like $\frac{\mu^{(r+1)}}{(r+1) !} \varepsilon^{r+1}$. To simplify further calculation, take $u=0$, since $\mu^{(r+1)}(0)$ is known, equal to $\alpha^{(r+1)}(0)$. In absolute value, the error is then comparable to $\lambda\left(\frac{\delta}{c n}\right)^{r+1}$. If this last quantity is set to be equal to $10^{-\eta}$, then a link appears between $r$ and $n$ :

$$
r=\frac{\eta \ln (10)+\ln (\lambda)}{\ln (c n / \delta)}-1, n=\frac{\delta}{c}\left(\lambda 10^{\eta}\right)^{1 /(r+1)} .
$$


For a given $u$ the local complexity of the algorithm is then proportional to

$$
c(r)=n r^{2}=\frac{\delta}{c} \lambda 10^{\eta \frac{1}{r+1}} r^{2} .
$$

Trying to find $r_{0}$ that minimizes $c(r)$, we find, in the case where $\ln \left(\lambda 10^{\eta}\right) \geq 8$

$$
r_{0}=\frac{1}{4}\left(\left(\ln \left(\lambda 10^{\eta}\right)-4+\sqrt{\ln \left(\lambda 10^{\eta}\right)\left(\ln \left(\lambda 10^{\eta}\right)-8\right)}\right), n_{0}=\frac{\delta}{c} \lambda 10^{\eta \frac{1}{r_{0}+1}} .\right.
$$

Since $n_{0}$ is here a real number, and should better be an integer greater than 1 , and since $r$ must also be an integer, we may choose the following parameters to ensure that required precision on $\mu$ is reached at the first point following $u=0$ :

$$
n_{\text {opt }}=\max \left(2,\left[n_{0}\right]+(0 \text { or } 1)\right) \text { and } r_{\text {opt }}=\left[\frac{\eta \ln (10)+\ln (\lambda)}{\ln \left(c n_{\text {opt }} / \delta\right)}\right]+(0 \text { or } 1) .
$$

As an example, take $\delta=100 \%$, so that we do not suppose that $\delta$ is close to 0 . For $\lambda=1, c=1$ and $\eta=12$ decimal digits, we get $n_{\text {opt }}=9$ and $r_{\text {opt }}=12$. With $\eta=16$ decimal digits, we get $n_{\text {opt }}=9$ and $r_{\text {opt }}=16$, so that the complexity is multiplied by something less than 1.8 to reach 4 more decimal digits.

\subsection{Bounds for $\mu_{u}, \mathrm{WF}(u, v)$ and their derivatives}

The algorithm makes only one approximation by replacing $\mu_{u+\varepsilon}$ with its Taylor expansion. Nevertheless, this approximation is used recursively, so that even if the error is locally bounded, we cannot ensure that the global result will be precise enough. For this reason, we must give exact bounds for the values we approximate.

For a function $f_{u}$ of $u$, we will use the following notations: $f_{u}{ }^{[-1]}$ and $f_{u}{ }^{[+1]}$ will be bounds of $f_{u}$, such that $f_{u} \in\left[f_{u}{ }^{[-1]}, f_{u}{ }^{[+1]}\right]$. We define by this way $\mu_{u}^{(k)[\sigma]}$, $S(u)^{[\sigma]}$ and $\beta_{u}^{(k)[\sigma]}$, with $\beta_{u}^{(k)}=\mathbb{E}\left[{ }_{W} p_{u-W}^{(k)}\right]$, for $\sigma \in\{-1,+1\}$.

For two bounded quantities $a$ and $b$, we will use following arithmetic, that might be simplified when the signs of $a$ and $b$ are known.

$$
\begin{aligned}
(a+b)^{[\sigma]} & =a^{[\sigma]}+b^{[\sigma]}, \\
(a-b)^{[\sigma]} & =a^{[\sigma]}-b^{[-\sigma]}, \\
(a b)^{[\sigma]} & =\max _{\sigma_{1}, \sigma_{2} \in\{-\sigma, \sigma\}}\left\{a^{\left[\sigma_{1}\right]} b^{\left[\sigma_{2}\right]}\right\}, \sigma \geq 0 \\
(a b)^{[-\sigma]} & =\min _{\sigma_{1}, \sigma_{2} \in\{-\sigma, \sigma\}}\left\{a^{\left[\sigma_{1}\right]} b^{\left[\sigma_{2}\right]}\right\}, \sigma \geq 0 .
\end{aligned}
$$

Note first that, when $u=0, S(0)^{[+1]}=S(0)^{[-1]}=1$. From (8), we can bound ${ }_{w} p_{u-w}^{(k)}$ from bounds of $\mu_{u}^{(j)}$ and ${ }_{w} p_{u-w}^{(j)}, j<k, w \leq u$. From (10), we can also 
bound $\mu_{u}^{(k)}$ from bounds of ${ }_{w} p_{u-w}^{(j)}, j \leq k$. We get then ${ }_{w} p_{u-w}^{(k)}{ }^{[\sigma]}$ and $\mu_{u}^{(k)[\sigma]}$ for $\sigma \in\{-1,+1\}$.

We will now use for a function $f_{u}$ of $u$ the following notations: $f_{u}{ }^{[-2]}$ and $f_{u}{ }^{[+2]}$ will be bounds of $f_{u}$, such that $\forall s<\varepsilon, f_{u+s} \in\left[f_{u}{ }^{[-2]}, f_{u}{ }^{[+2]}\right]$.

Note that, when $u=0, S(0)^{[+2]}=1$, and since $S$ is decreasing, $S(0)^{[-2]} \leq$ $S(\varepsilon)^{[-1]}$. The sign of $\alpha_{u}^{(k)}$ is the same as the one of $(-1)^{k}$. Since $\alpha_{u}^{(k)}$ is thus either increasing or decreasing in $u$, depending on $k \bmod 2$, we remark that

$$
\begin{aligned}
\alpha_{u}^{(k)[\sigma]} & =k ! \lambda(-\delta)^{k}(c+\delta u)^{-(k+1)}, \sigma=-1,0,+1, \\
\alpha_{u}^{(k)[-2]} & =\mathbf{1}_{\{k \bmod 2=0\}} \alpha_{u+\varepsilon}^{(k)}+\mathbf{1}_{\{k \bmod 2=1\}} \alpha_{u}^{(k)}, \\
\alpha_{u}^{(k)[+2]} & =\mathbf{1}_{\{k \bmod 2=0\}} \alpha_{u}^{(k)}+\mathbf{1}_{\{k \bmod 2=1\}} \alpha_{u+\varepsilon}^{(k)} .
\end{aligned}
$$

Using such $\alpha_{u}^{(k)[\sigma]}$, we can easily check that (8) and (10) can be adapted to get bounds ${ }_{w} p_{u-w}^{(k)}{ }^{[\sigma]}$ and $\mu_{u}^{(k)[\sigma]}$ for $\sigma \in\{-2,+2\}$.

The knowledge of bounds of $\mu_{u+s}^{(k)}, s<\varepsilon$ will allow us to derive bounds of the derivative form of Taylor's remainder, and then bounds of $S(u)$. For $s \in[0, \varepsilon[$, $\mu_{u+s}$ is a continuous and $r+1$ times differentiable function of $s$. We have

$$
\mu_{u+s}=\sum_{k=0}^{r} \frac{\mu_{u}^{(k)}}{k !} s^{k}+R_{u, s}^{(r)}, \text { with } R_{u, s}^{(r)}=\frac{\mu_{u^{*}}^{(r+1)}}{(r+1) !} s^{r+1}, u^{*} \in[u, u+\varepsilon[.
$$

Since $\mu_{u *}^{(r+1)}$ is bounded, we can bound $R_{u, s}^{(r)}$, and then $S(u+\varepsilon)$ :

$S(u+\varepsilon)^{[\sigma]}=S(u)^{[\sigma]} \exp \left(-\sum_{k=0}^{r} \frac{\mu_{u}^{(k)[-\sigma]}}{(k+1) !} \varepsilon^{k+1}+\frac{\mu_{u}^{(r+1)[-2 \sigma]}}{(r+2) !} \varepsilon^{r+2}\right), \sigma \in\{-1,+1\}$.

The only difficulty to build the bounding algorithm is the following: since $S(u)$ is decreasing in $u$, a good lower bound for $S(x), x \in[u, u+\varepsilon[$ is given by the lowest value of $S(u+\varepsilon)$, so that we can propose $S(u)^{[-2]}=S(u+\varepsilon)^{[-1]}$. Nevertheless, the calculation of $S(u+\varepsilon)^{[-1]}$ from $S(u)^{[-1]}$ uses $\mu_{u}^{(r+1)[+2]}$, that is then calculated from $S(u)^{[-2]}$. Using such a bound gives then $S(u)^{[-2]}$ as a computable function of itself. We have built both a formal computation algorithm, in order to get the root value of $S(u)^{[-2]}$, and also a fixed-point algorithm, starting from $S(u)^{[-2]}=0$. Nevertheless, since the last term of Taylor expansion becomes very small for large values of $r$, such precise bounds of $S(u)^{[-2]}$ could be replaced with $S(u)^{[-2]}=0$. The great acceleration resulting of this choice can be exploited to increase $r$ or $n=1 / \varepsilon$, for example, and thus the precision of the algorithm. We will see with numerical figures that this approximation is sufficient to get very precise results. Indeed, it only changes bounds for the $r+1^{\text {th }}$ derivative order of $\mu_{u}$ and has an impact comparable to $\mu_{u}^{(r+1)[+2]} \varepsilon^{r+2} /(r+2)$ !. The problem does not hold for $S(u)^{[+2]}$ since the better bound we can propose is $S(u)^{[+2]}=S(u)^{[+1]}$. Note that, by construction, 
$S(u)^{[-1]}$ and $S(u)^{[+1]}$ give bounds for $S(u)$, not for its approximation $S(u)^{[0]}$, which can be outside the interval.

Proposition 5 (bounding algorithm) Bounds for $\mu_{u}, \mathbb{E}\left[{ }_{W} p_{u-W}\right], S(u)$ and their derivatives up to order $r$ are given by following algorithm, with initialization values $S(0)^{[-1]}=S(0)^{[+1]}=1$. For $u=0 . . u_{\max }$ by step $\varepsilon$, for $k=0 . . k+1$, and for $\sigma_{0}=+1,+2$,

$$
\begin{aligned}
& S(u)^{[+2]}=S(u)^{[+1]}, S(u)^{[-2]}=0 \\
& { }_{w} p_{u-w}^{(0)}{ }^{[\sigma]}=\frac{S(u)^{[\sigma]}}{S(u-w)^{[-\sigma]}}, \sigma= \pm \sigma_{0} \\
& { }_{w} p_{u-w}^{(k)}{ }^{[\sigma]}=\sum_{j=0}^{k-1} C_{k-1}^{j}\left({ }_{w} p_{u-w}^{(k-1-j)}\left(\mu_{u-w}^{(j)}-\mu_{u}^{(j)}\right)\right)^{[\sigma]}, u \geq 1, k \geq 1, \sigma= \pm \sigma_{0} \\
& \mu_{u}^{(k)[\sigma]}=\alpha_{u}^{(k)[\sigma]}-\mathbf{1}_{\{u \geq 1\}} \sum_{j=0}^{k} C_{k}^{j}\left(\alpha_{u}^{(k-j)} \mathbb{E}\left[W p_{u-W}^{(j)}\right)^{[-\sigma]}, \sigma= \pm \sigma_{0}\right. \\
& S(u+\varepsilon)^{[\sigma]}=S(u)^{[\sigma]} \exp \left(-\sum_{k=0}^{r} \frac{\mu_{u}^{(k)[-\sigma]}}{(k+1) !} \varepsilon^{k+1}-\frac{\mu_{u}^{(r+1)[-2 \sigma]}}{(r+2) !} \varepsilon^{r+2}\right), \sigma= \pm 1 .
\end{aligned}
$$

This algorithm is quite similar to the first one we proposed. Some remarks can be done for its practical implementation.

First, we had better use only integer arguments, so that for $n=1 / \varepsilon, n \in \mathbb{N}$, we preferably replace $u$ with an index $i=0 . . n u_{\max }$, where $i$ denotes $n u$.

Second, for each value of $u$, we do not use previous values ${ }_{w} p_{u_{0}-w}^{(k)}$ and $\mathbb{E}\left[{ }_{W} p_{u_{0}-W}^{(k)}\right]$, $u_{0}<u$. In the algorithm, these quantities do not need to depend on $u$, and that spares stocking memory.

Third, many quantities, like $\mathbb{E}\left[{ }_{W} p_{u-W}^{(k)}\right]$ or like Taylor integrated approximation in the exponential, can be computed in previous sums giving respectively ${ }_{w} p_{u-w}^{(k)}$ and $\mu_{u}^{(k)}$.

We may check at each step if the precision of the computer is high enough. If not, it is possible to change lower and upper bounds in order to include, at each step, the maximum numerical computer error.

At last, bounds for derivatives of $S$ and $\operatorname{WF}(u, v)$ with respect to $u$ and $v$ are given for $\sigma \in\{-2,-1,1,2\}$ by:

$$
\begin{aligned}
S^{(k+1)[\sigma]}(x) & =-\sum_{i=0}^{k} C_{k}^{i}\left(\mu_{x}^{(i)} S^{(k-i)}(x)\right)^{[\sigma]} . \\
\frac{\partial^{k}}{\partial v^{k}}{ }^{[\sigma} p_{u}{ }^{[\sigma]} & =S^{(k)}(u+v)^{[\sigma]} / S(u)^{[-\sigma]}, k \geq 0, \\
\frac{\partial^{k}}{\partial u^{k}}{ }^{[-\sigma} p_{u}{ }^{[\sigma]} & =\sum_{i=0}^{k-1} C_{k-1}^{i}\left({ }_{v} p_{u}^{(k-1-i)}\left(\mu_{u}^{(i)}-\mu_{u+v}^{(i)}\right)\right)^{[\sigma]}, k \geq 1 .
\end{aligned}
$$




\subsection{Further results and improved algorithm}

We have seen that, given the survival function $S(x)$ for $x \in[0, u]$, it is possible to deduce exactly as many derivatives of $\mu_{u}$ and ${ }_{w} p_{u-w}$ as wanted, and to get then an approximation of $S(u+\varepsilon)$. The previous algorithm was constructed on this idea. For $k$ varying from 0 to a given derivative order $r$, let us recall here equations that are used in this exact differentiation step $(w \leq u)$ :

$$
\begin{aligned}
{ }_{w} p_{u-w}^{(k)} & =\mathbf{1}_{\{k=0\}} \frac{S(u)}{S(u-w)}+\mathbf{1}_{\{k \geq 1\}} \sum_{i=0}^{k-1} C_{k-1 w}^{i} p_{u-w}^{(i)}\left(\mu_{u-w}^{(k-1-i)}-\mu_{u}^{(k-1-i)}\right) \\
\mu_{u}^{(k)} & =\alpha_{u}^{(k)}-\sum_{j=0}^{k} C_{k}^{j} \alpha_{u}^{(k-j)} \mathbb{E}\left(W p_{u-W}^{(j)}\right) \\
\alpha_{u}^{(k)} & =k ! \frac{\lambda}{c+\delta u}\left(\frac{-\delta}{c+\delta u}\right)^{k}
\end{aligned}
$$

This step was of complexity proportional to $u r^{2}$. We will see here that it is sometimes possible to reduce this complexity to something proportional to $\ln (u) r^{2}$. To do so, we shall denote by $\Omega_{\nu}$ a random variable distributed as $W^{* 2^{\nu}}=W_{1}+\ldots+W_{2^{\nu}}$, with $\Omega_{0}=W$. The law of $\Omega_{\nu}$ can be easily constructed for integer claim amount $W$, since for $k \in \mathbb{N}$,

$$
\begin{aligned}
\mathbb{P}\left[\Omega_{0}=k\right] & =\mathbb{P}[W=k], \\
\mathbb{P}\left[\Omega_{\nu+1}=k\right] & =\sum_{i=0}^{k} \mathbb{P}\left[\Omega_{\nu}=i\right] \mathbb{P}\left[\Omega_{\nu}=k-i\right], \quad k \in \mathbb{N}, \nu \geq 0 .
\end{aligned}
$$

Remark also that if $S$ is given on $[0, u]$, we can easily deduce ${ }_{\Omega_{\nu}} p_{u-\Omega_{\nu}}$ from $S$. We will see that since $W \geq 1$ we will only need law of $\Omega_{\nu}$ when $\Omega_{\nu} \leq u$, i.e. for $\nu \leq \frac{\ln (u)}{\ln (2)}$.

We previously gave derivatives for almost all relations, except an important one:

Proposition 6 By derivation of actuarial property of win-first probabilities,

$$
\begin{aligned}
{ }_{s+t} p_{x} & ={ }_{s} p_{x} \cdot{ }_{t} p_{x+s}, \\
{ }_{s+t} p_{x}^{(k)} & =\sum_{j=0}^{k} C_{k s}^{j} p_{x}^{(j)}{ }_{t} p_{x+s}^{(k-j)} .
\end{aligned}
$$


Consider first the case $\delta=0$. In this case, $\alpha_{u}^{(k)}=\frac{\lambda}{c} \mathbf{1}_{\{k=0\}}$. As ${ }_{w} p_{u-w}=0$ when $w>u$, injecting (15) into (14) gives ${ }_{W_{1}} p_{u-W_{1}}^{(k+1)}=\frac{\lambda}{c} \sum_{i=0}^{k} C_{k W_{1}}^{i} p_{u-W_{1}}^{(i)} \mathbb{E}_{W_{2}}\left[W_{2} p_{u-W_{2}}^{(k-i)}\right]-\frac{\lambda}{c} \sum_{i=0}^{k} \mathbb{E}_{W_{2}}\left[C_{k W_{2}}^{i} p_{u-W_{1}-W_{2} W_{1}}^{(k-i)} p_{u-W_{1}}^{(i)}\right]$

Using proposition 6 , we get then the following theorem, reducing the complexity of differentiation step to something proportional to $\ln (u) r^{2}$ :

Theorem 6 When $\delta=0$, and if $S(x)$ is given on $x \in[0, u]$, then all derivatives of $\Omega_{\nu} p_{u-\Omega_{\nu}}$ are given by the following recursion: for $\nu$ from $[\ln u / \ln 2]$ down to 0 , for $k$ from 0 to $r$,

$$
\begin{aligned}
\beta_{u, \nu}^{(k+1)} & =\mathbf{1}_{\left\{2^{\nu} \leq u\right\}}\left(-\frac{\lambda}{c} \beta_{u, \nu+1}^{(k)}+\frac{\lambda}{c} \sum_{i=0}^{k} C_{k}^{i} \beta_{u, \nu}^{(i)} \beta_{u, \nu}^{(k-i)}\right), k \geq 0, \delta=0 \\
\text { with } \beta_{u, \nu}^{(k)} & =\mathbb{E}\left[\Omega_{\nu} p_{u-\Omega_{\nu}}^{(k)}\right] .
\end{aligned}
$$

Consider now the case $\delta>0$. In this case, note that

$$
C_{k}^{j} \alpha_{u}^{(j)} \alpha_{u}^{(k-j)}=\alpha_{u}^{(0)} \alpha_{u}^{(k)}, \text { with } \alpha_{u}^{(0)}=\frac{\lambda}{c+\delta u} .
$$

Assume that $u$ is given and define $\tilde{\mu}_{x}^{(k)}=\mu_{x}^{(k)} / \alpha_{u}^{(k)},{ }_{t} \tilde{p}_{x}{ }^{(k)}={ }_{t} p_{x}^{(k)} / \alpha_{u}^{(k)}$ and $\tilde{\alpha}_{x}^{(k)}=\alpha_{x}^{(k)} / \alpha_{u}^{(k)}$. Substituting (18) in (14) and (15) yields:

$$
\begin{aligned}
{ }_{w} \tilde{p}_{x-w}^{(k+1)} & =\gamma_{k} \sum_{i=0}^{k}{ }_{w} \tilde{p}_{x-w}^{(i)}\left(\tilde{\mu}_{x-w}^{(k-i)}-\tilde{\mu}_{x}^{(k-i)}\right), \text { with } \gamma_{k}=\frac{-\lambda}{(k+1) \delta}, \\
\tilde{\mu}_{x}^{(k)} & =\tilde{\alpha}_{x}^{(k)}-\alpha_{u}^{(0)} \sum_{j=0}^{k} \tilde{\alpha}_{x}^{(k-j)} \mathbb{E}\left({ }_{W} \tilde{p}_{x-W}^{(j)}\right),
\end{aligned}
$$

and from 17,

$$
{ }_{s+t} \tilde{p}_{x}^{(k)}=\frac{\lambda}{c+\delta u} \sum_{j=0}^{k}{ }_{s} \tilde{p}_{x}^{(j)}{ }_{t} \tilde{p}_{x+s}^{(k-j)} .
$$

Equations (19) to (21) could be useful for computations and for further analysis, since they avoid to compute binomial terms in the recurrence or factorials in the Taylor's expansion:

$$
\sum_{k=0}^{r} \frac{\mu_{u}^{(k)}}{(k+1) !} \varepsilon^{k+1}=\frac{\lambda \varepsilon}{c+\delta u} \sum_{k=0}^{r} \frac{\tilde{\mu}_{u}^{(k)}}{k+1}\left(\frac{-\delta \varepsilon}{c+\delta u}\right)^{k}
$$


This improvement being quite simple, the resulting algorithms for approximation and bounds are omitted here.

Other extensions may be found for $\delta>0$ by similar arguments as in the case $\delta=0$. Injecting (20) into (19), and using the actuarial property, we can get an expression depending on quantities $\tilde{\alpha}_{u-w}^{(k)}, w \leq u$. Since these quantities are bounded, with $\tilde{\alpha}_{u-w}^{(k)} \in\left[1,\left(1+\delta \frac{u}{c}\right)^{k+1}\right]$, we can derive recursive bounds for $\tilde{\beta}_{u, \nu}^{(k)}=\mathbb{E}\left[\Omega_{\nu} \tilde{p}_{u-\Omega_{\nu}}^{(k)}\right]$ as a function of $\tilde{\beta}_{u, \nu+1}^{(k)}$, for $k \geq 0$ and $\delta>0$. We can thus construct bounds for $\tilde{\beta}_{u}=\beta_{u, 0}^{(k)}, \tilde{\mu}_{u}^{(k)}$ and $\mu_{u}^{(k)}$. The complexity of the differentiation step is then proportional to $\ln (u) r^{2}$ instead of $u r^{2}$, but the obtained bounds are less precise than in previous bounding algorithm. Nevertheless, this approach might be useful when looking for analytic bounds of $\tilde{\beta}_{u, \nu}^{(k)}$.

\section{Applications and numerical results}

\subsection{An example of application : payment of dividends}

Let us now modify our process $R_{t}$ with an horizontal dividend barrier strategy. Starting from $u$, if the surplus reaches the upper barrier $u+v$, all the premium income and the interests (at rate $\delta$ ) are paid as dividends until the next claim, i.e. during an exponentially distributed time $\xi$, with parameter $\lambda$. We shall show here that it is possible to determine the total amount of dividends that will be paid until the process reaches the lower barrier 0 , and that this cumulative amount of dividends depends on win-first probabilities and on quantities which are computed in the previous algorithm. The total expected amount of dividends is given here as a simple example, and depending on the purpose of the study, one may introduce either a discounting factor or other parameters. We shall keep in mind that the total dividend amount might be here represented by a defective random variable. Denote by $D_{i}$ the cumulative amount of dividends that is paid during the $i^{\text {th }}$ period of payment, distributed as $D=\int_{0}^{\xi} e^{\delta s} d s$, where is $N$ the number of payment periods, and $T$ is the total amount of dividends $T=\sum_{i=0}^{N} D_{i}$. We also use $N_{0}$ and $T_{0}$, the random variables distributed as $N$ and $T$ given that $N>0$. For any random variable $X$, we will denote respectively by $F_{X}$ and $f_{X}$ its distribution and density function. From the memoryless property of the modified risk process, $N_{0}-1$ is a geometric random variable with parameter $\beta_{u+v}=\mathbb{E}[\operatorname{WF}(u+v-W, W)]$, and it is easy to get the classical results :

$$
\mathbb{P}[N=0]=1-\mathrm{WF}(u, v) \quad \text { and } \quad \mathbb{P}[N=k]=\mathrm{WF}(u, v) \beta_{u+v}^{k-1}\left(1-\beta_{u+v}\right), k \geq 1,
$$

and if $\lambda>\delta$ and $\beta_{u+v}<1$, supposing that $u$ and $v$ are fixed, with the notation $\omega=\mathrm{WF}(u, v)$ and $\beta=\beta_{u+v}$, the distribution function and the mean of the cumulated amount of dividends are as follows : 


$$
\begin{aligned}
\mathbb{P}[T \leq x] & =(1-\omega)+\omega(1-\beta) \sum_{k=1}^{\infty} \beta^{k-1} F_{D^{* k}}(x), \\
\mathbb{E}[T] & =\frac{\mathrm{WF}(u, v)}{\left(1-\beta_{u+v}\right)(\lambda-\delta)} .
\end{aligned}
$$

The expected value only depends on $\lambda, \delta, \mathrm{WF}(u, v)$ and $\beta_{u+v}$, which we are able to compute with as much precision as necessary.

Proposition 7 If there exists $R$ such that $\mathbb{E}\left[e^{R D}\right]=1 / \beta$, then we can get, by application of Smith's theorem,

$$
\lim _{x \rightarrow \infty} \mathbb{P}[T \leq x]=(1-\omega)+\omega \lim _{x \rightarrow \infty} e^{-R x} \frac{\int_{0}^{\infty} \bar{a}(y) d y}{\int_{0}^{\infty}(1-\bar{G}(y)) d y}
$$

with $\bar{a}(y)=(1-\beta) e^{R y} F_{D}(y), d \bar{G}(y)=\beta e^{R y} f_{D}(y), F_{D}(y)=1-(1+\delta y)^{-\frac{\lambda}{\delta}}$, and $f_{D}(y)=\lambda(1+\delta y)^{\left(-\frac{\lambda}{\delta}-1\right)}$.

This simple example shows how quantities $\beta=\mathbb{E}[\mathrm{WF}(u+v-W, W)]$ and $\omega=\mathrm{WF}(u, v)$ naturally appear in the computation of the expected dividends.

\subsection{Numerical results}

The results presented hereafter have been obtained for $\lambda=1$ and $c=1.05 . \mathrm{W}$ is first exponentially distributed with parameter 1 , and then discretized with $F_{d}(i d)$ defined on each interval $[i d, i d+d[$, such that:

$$
F_{d}(i d)=\frac{1}{d} \int_{[i d, i d+d[} F_{W}(x) d x
$$

We have taken $d=1$. As explained in section 3.1, in order to cancel $\pi_{0}=$ $\mathbb{P}(W=0)$, the Poisson parameter $\lambda$ has been modified into $\lambda\left(1-\pi_{0}\right)$, and the $\pi_{i}$ have been changed too. This discretization procedure if fully described in De Vylder (1999). This explains values for $x=0$ in figures 2 and 3. The observation of the evolution of $\mu_{x}, x>0$ for integer-valued claim amounts confirm that it is nonincreasing, but not continuous. This is a classical fact in ruin theory, and it explains that we usually observe discontinuity points that really exist, even if exact computations are carried out. Let us explain, for example, that if $\delta=0, \mu_{x}=\mu_{0}$ for each $x<1$. Note that $\theta=\sup \left\{R_{t}, t \leq T_{0} \mid R_{0}=0\right\}$. Starting from 0 , the random variable $\theta$ keeps growing, as a survival lifetime, until the first claim. If the claim occurs before $\theta$ reaches the value 1 , then ruin occurs since the claim amount is a positive integer. As long as $\theta<1$, for $\delta=0$, the probability that $\theta$ stops growing is directly linked with the hazard rate of the time of the first claim, which is constant and equal to the modified $\lambda$. If 


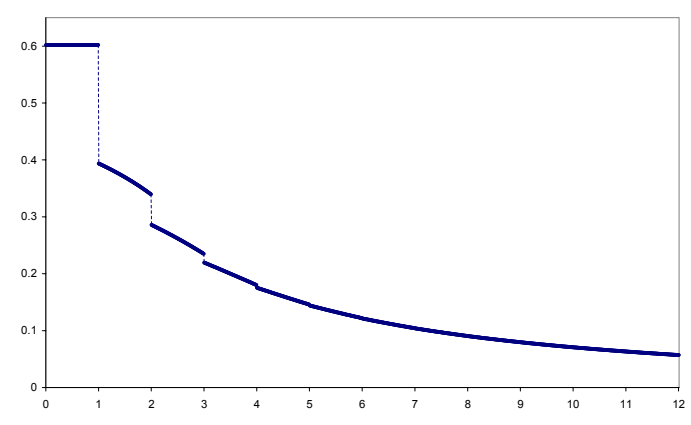

Figure 2. Aspect of $\mu$ for integer-valued $W$ and $\delta=0$.

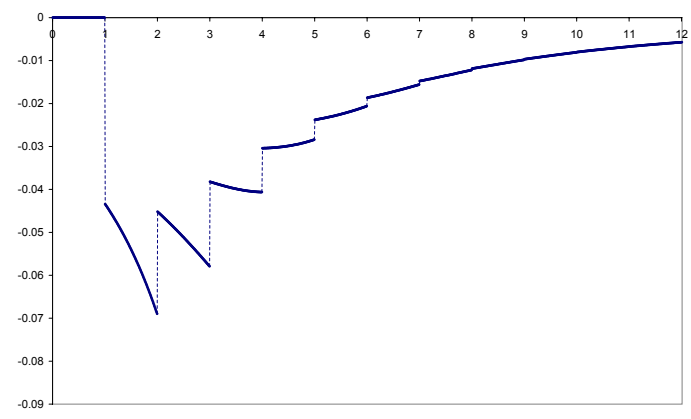

Figure 4. Aspect of derivative function of hazard rate $\mu_{x}^{\prime}, x \notin \mathbb{N}$ and $\delta=0$.

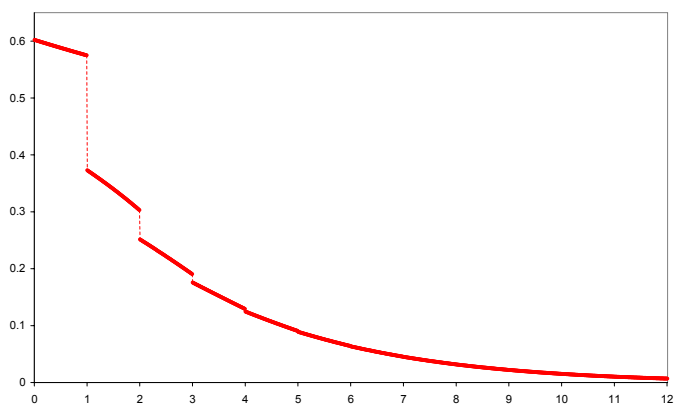

Figure 3. Aspect of $\mu$ for integer-valued $W$ and $\delta=0.05$.

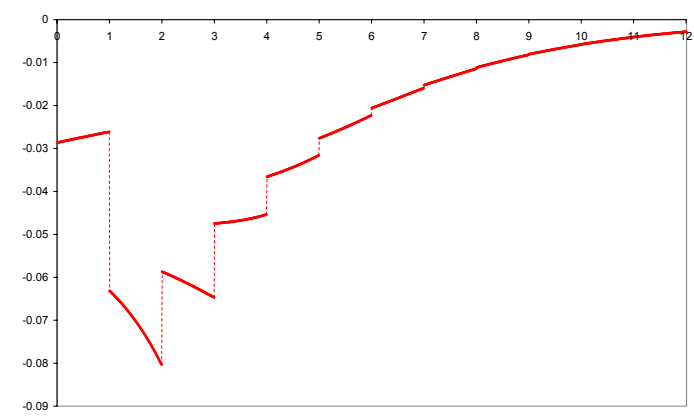

Figure 5. Aspect of derivative function of hazard rate $\mu_{x}^{\prime}, x \notin \mathbb{N}$ and $\delta=0.05$.

$\theta$ has reached $x>1$, situation is more complex, since the probability that $\theta$ stops growing will also depend on the claim amount.

The analysis of derivatives of hazard rates (see figures 4 and 5) may be important to understand approximations that are made in the proposed algorithm. Replacing $\mu_{i \varepsilon}^{(k+1)}$ with the approximation $\mu_{i \varepsilon}^{\text {left }(k)}=\left(\mu_{i \varepsilon}^{(k)}-\mu_{(i-1) \varepsilon}^{(k)}\right) / \varepsilon$ gives good results for $i \varepsilon \notin \mathbb{N}$, but it must be done keeping in mind the discontinuity of $\mu_{x}$ and of its derivatives on atoms of the distribution of the claim amount (see tables 1 and 2). Despite discontinuities of hazard rates of $\theta$ (see figures 2 and 3), survival function $S(x)$ is continuous (see figure 6), and tends to $\varphi_{\delta}(0)$ as $x \rightarrow+\infty$. This function is sufficient to obtain all values of $\mathrm{WF}(u, v)=S(u+v) / S(u), u, v>0$. Of course, in the special case $\delta=0$, computation of probabilities of ruin and non-ruin are already well-known, and may be computed for example with classical formulae (see Picard and Lefèvre (1997) or Rullière and Loisel (2004)). We retrieve $S(u)$ by computing the ratio $\varphi_{0}(0) / \varphi_{0}(u)$ (see table 3 ). We shall remember that, for $u>0$, although the computation of $\varphi_{0}(u)$ is exact, it does not use previous computations of $\varphi_{0}(x), x=1, . . u-1$. This implies, especially if discretization of $W$ is really accurate, a computation time that could be important. It is thus interesting to propose another way to determine $\mathrm{WF}(u, v)$ that would help to understand 


\begin{tabular}{|l|c|r|}
\hline$i \varepsilon$ & $\mu_{i \varepsilon}^{(1)}$ & $\mu_{i \varepsilon}^{\prime l e f t}$ \\
\hline 4.95 & -0.02858 & -0.02860 \\
4.96 & -0.02854 & -0.02856 \\
4.97 & -0.02850 & -0.02852 \\
4.98 & -0.02846 & -0.02848 \\
4.99 & -0.02842 & -0.02844 \\
$\mathbf{5}$ & $\mathbf{- 0 . 0 2 3 8 0}$ & $\mathbf{- 0 . 1 7 0 4 1}$ \\
5.01 & -0.02378 & -0.02379 \\
5.02 & -0.02376 & -0.02377 \\
\hline
\end{tabular}

Table 1 Some values of derivatives of $\mu$ for $\delta=0$ and $\varepsilon=0.01$.

\begin{tabular}{|l|c|r|}
\hline$i \varepsilon$ & $\mu_{i \varepsilon}^{(1)}$ & $\mu_{i \varepsilon}^{\prime l e f t}$ \\
\hline 4.95 & -0.03185 & -0.03188 \\
4.96 & -0.03179 & -0.03182 \\
4.97 & -0.03173 & -0.03176 \\
4.98 & -0.03166 & -0.03169 \\
4.99 & -0.03160 & -0.03163 \\
$\mathbf{5}$ & $\mathbf{- 0 . 0 2 7 6 3}$ & $\mathbf{- 0 . 1 6 9 3 6}$ \\
5.01 & -0.02758 & -0.02761 \\
5.02 & -0.02753 & -0.02756 \\
\hline
\end{tabular}

Table 2 Some values of derivatives of $\mu$ for $\delta=0.05$ and $\varepsilon=0.01$.

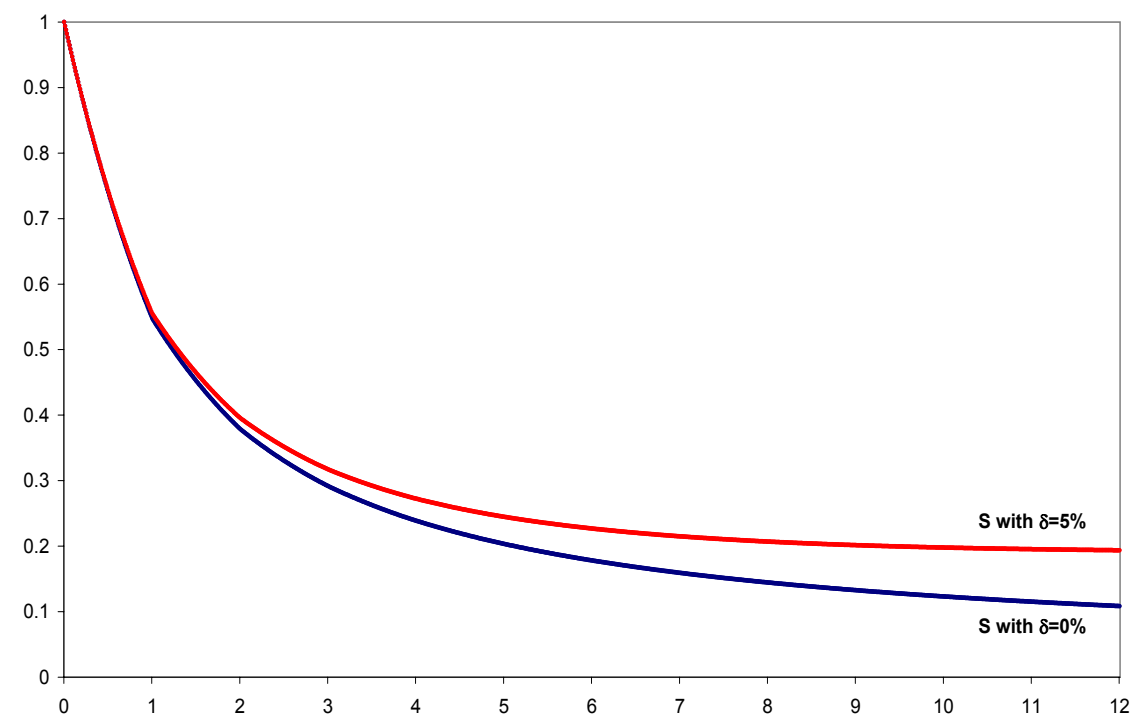

Figure 6. Aspect of survival function $S(u)=\mathrm{WF}(0, u)$ for $\delta=0$ and $\delta=0.05$.

the structure of $\theta$. In table 3 , we see that approximation algorithm gives quite precise results for small values of convergence parameters $n$ and $r$, and that precision increases rapidly when $r$ becomes larger.

To give an idea of the convergence of the bounding algorithm, we have taken convergence parameters $n=2$ and $r=100$. Keeping $c=1.05, \lambda=1$, we obtain quantities in tables $4,5,6,7$ and 8 , in both cases $\delta=0.05$ or $\delta=1.2$. Rather than proposing very near bounds for each quantity, we preferred showing only decimals that were in common in lower and upper bounds. The great number of correct digits shows that the algorithm gives very thin bounds when $r$ becomes large. It may help measuring quality of analytical approximations, and also helps comparing precision of the algorithm with the existing one in the literature. This last point will be developed in section 4.3. In table 6, we gives bounds for the 10 first order derivatives of $S$ and $\mu$. When $r$ is large enough, bounds remain very thin also for these derivatives, and are far much precise than the one that could be obtained by successive finite differences on 


\begin{tabular}{|c|c|c|c|c|}
\hline$u$ & $\varphi_{0}(u)$ & $\varphi_{0}(0) / \varphi_{0}(u)$ & $S(u)_{(n=2, r=2)}$ & $S(u)_{(n=2, r=7)}$ \\
\hline 0 & 0.047619048 & 1 & 1 & 1 \\
1 & 0.086942973 & 0.547704386 & .5477043856 & .5477043856 \\
2 & 0.125654634 & 0.378967699 & .3789347571 & .3789676986 \\
3 & 0.163135685 & 0.291898413 & .2918589855 & .2918984132 \\
4 & 0.199174553 & 0.239081985 & .2390475932 & .2390819852 \\
5 & 0.233726482 & 0.203738350 & .2037113041 & .2037383494 \\
6 & 0.266813025 & 0.178473475 & .1784528790 & .1784734745 \\
7 & 0.298480705 & 0.159538110 & .1595224001 & .1595381102 \\
8 & 0.328784306 & 0.144833700 & .1448214757 & .1448336999 \\
9 & 0.357780267 & 0.133095791 & .1330859961 & .1330957906 \\
10 & 0.385524138 & 0.123517681 & .1235095719 & .1235176811 \\
\hline
\end{tabular}

Table 3

Exact values of $\varphi_{0}(u)$ by the Picard-Lefèvre formula and approximations of $S(u)$ $(\delta=0)$.

\begin{tabular}{|c|c|c|}
\hline$u$ & $S(u)$ & $\mu_{u}$ \\
\hline 0 & 1. & .60201957983672159848045355222718012624208463711260 \\
1 & .55536753143898948033704731623796351501937756358898 & .37291709040266536547962244132582576831761412574029 \\
2 & .39571061661657290808739908681011144489297103305312 & .25151862906429592201939126794787995307626601247085 \\
3 & .31717796643173124672644036387953769101531776076477 & .17561721629275419932396285268627620586636125028427 \\
4 & .27241949864280665916591655760301981552636588391855 & .12463074207617205987829396215227588738956312457840 \\
5 & .24475728269819191947606478608113230139779082228707 & .088983882528775672523830328545016100434528133542912 \\
6 & .22684151014642003046567015318789865261680613384077 & .063489602807296175860368607790255643020687513766155 \\
7 & .21492640570772406604762219996119093708403247013515 & .045055660722929856034785799235531431265782502796578 \\
8 & .20689527993852467459282972172931079384070536660447 & .031695402749427521457236730930928679745933711961554 \\
9 & .20145762751247551497216845937090037598532454531247 & .022050837923035677891605896212785430522573468958071 \\
10 & .19778202146032724398088007977799747009305673079175 & .015147834698460297368806746234185068943442859266538 \\
\hline
\end{tabular}

Table 4

Exact decimal digits of $S(u)$ and $\mu_{u}$ by bounding algorithm $(\delta=0.05)$.

Table 5

\begin{tabular}{|c|c|c|}
\hline$u$ & $S(u)$ & $\mu_{u}$ \\
\hline 0 & 1. & .60201957983672159848045355 \\
1 & .66933517879990261091566493 & .16207556315205895533649587 \\
2 & .59730976442093773603118302 & .05441722349579981821432819 \\
3 & .57494353077840354664777459 & .01938689080540239232950103 \\
4 & .56725683117542653295887268 & .00703790102742977713159014 \\
5 & .56452041446391119585445702 & .00257056467920173233831039 \\
6 & .56353310298614385390522442 & .00094069917249684588476497 \\
7 & .56317486756630610711255072 & .00034444955377647221733089 \\
8 & .56304453577548005640595134 & .00012614794861816208792551 \\
9 & .56299704696737238590046541 & .00004620339806683924991726 \\
10 & .56297972609520189881409116 & .00001692406622986125300514 \\
\hline
\end{tabular}

Exact decimal digits for $S(u)$ and $\mu_{u}$ by bounding algorithm $(\delta=1.2)$. 


\begin{tabular}{|c|c|c|}
\hline$k$ & $S(u)^{(k)}$ & $\mu_{u}^{(k)}$ \\
\hline 0 & .244757282698191919476064786081132301397790822287 & .088983882528775672523830328545016100434528133542 \\
1 & -.021779453291678248139853343964504022264773000051 & -.027629483673356369324950664995212902914462559697 \\
2 & .008700537659492416889348321750556619101482134957 & .004781759780650901141178540920815767739921848509 \\
3 & -.003148088249731182436118591683531570999288924430 & .001184056174728555633066782660597992807695785290 \\
4 & .001023929174136961425000957228656082809071769453 & .000079184281921029225092433587328484379390687953 \\
5 & -.000604885236309820279361901997938225188501618568 & -.000402623545415252800623317732679845596127644524 \\
6 & .000349960969975695293643389220473108020035197533 & -.000386370000451382731645846489531581540120338400 \\
7 & -.000098690210126223893537006123193270538997311567 & -.000179180225484452079551481731074750159874505135 \\
8 & .000162013429988748369733435193279071491692638059 & .000076722640836791688533098696105266989982210445 \\
9 & -.000075506148302808719759688754740222191140782554 & .000264991262234335480487532290438883180373071878 \\
10 & -.000008701456970729554583982507829971722968348809 & .000285704192102520471952411764658858649021463062 \\
\hline
\end{tabular}

Table 6

Exact decimal digits of right derivatives $S(u)^{(k)}$ and $\mu_{u}^{(k)}(u=5, \delta=0.05)$.

Table 7

\begin{tabular}{|c|c|}
\hline quantity & value for $u=5, v=4, \delta=0.05$ \\
\hline $\mathrm{WF}(u, v)$ & $.8230914532618470298053719011486982427784142006271074 \ldots$ \\
$\frac{\partial}{\partial u} \mathrm{WF}(u, v)$ & $.0550920169557785632478932959551552772017674284516602 \ldots$ \\
$\frac{\partial}{\partial v} \mathrm{WF}(u, v)$ & $-.01814985623171288470150842179406380159358909721724867 \ldots$ \\
$\mathbb{E}[\mathrm{WF}(u-W, W)]$ & $.816998441718484612223534760053005148559101965324086718334 \ldots$ \\
$\lambda /(c+\delta u)$ & $.486246583714275137234212484491183948118606822283255511917 \ldots$ \\
\hline
\end{tabular}

Exact decimal digits of quantities in differential equation by bounding algorithm $(\delta=0.05)$.

Table 8

\begin{tabular}{|c|c|}
\hline quantity & value for $u=5, v=4, \delta=1.2$ \\
\hline $\mathrm{WF}(u, v)$ & $.9973014837771890777610838 \ldots$ \\
$\frac{\partial}{\partial u} \mathrm{WF}(u, v)$ & $.00251754925126551481612193 \ldots$ \\
$\frac{\partial}{\partial v} \mathrm{WF}(u, v)$ & $-.000046078717447606893398087 \ldots$ \\
$\mathbb{E}[\mathrm{WF}(u-W, W)]$ & $.97133065720571295060131280 \ldots$ \\
$\lambda /(c+\delta u)$ & $.08966249061397981253964201841681406 \ldots$ \\
\hline
\end{tabular}

Exact decimal digits of quantities in differential equation by bounding algorithm $(\delta=1.2)$.

thin intervals of length $\varepsilon$. This result comes directly from the fact that (5) gives at point $x$ exact values of these derivatives, when $S$ is given on $[0, x]$. Only approximations on $S$, that are numerically very precise, have an impact on these derivatives. At last, we have bounded, for $u=5$ and $v=4$, terms appearing in differential equation (3):

$$
\frac{\partial}{\partial u} \mathrm{WF}(u, v)-\frac{\partial}{\partial v} \mathrm{WF}(u, v)=\frac{\lambda}{c+\delta u} \mathrm{WF}(u, v) \cdot(1-\mathbb{E}[\mathrm{WF}(u-W, W)]) .
$$

Only decimals that are in common in lower and upper bounds are written in tables 7 and 8 . As $\lambda$ was modified to eliminate the mass $P[W=0]$, we easily verify this differential equation, in both cases $\delta=0.05$ and $\delta=1.2$. Convergence parameters $n=2$ and $r=100$ give in both cases, for the equality, a 


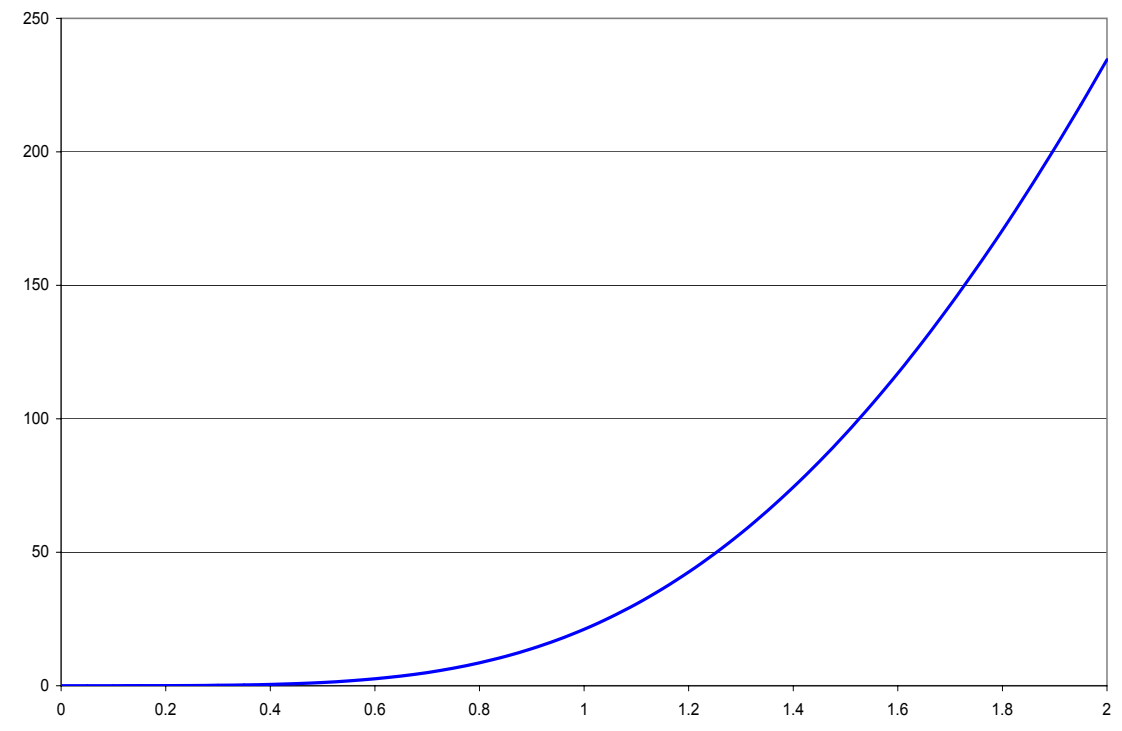

Figure 7. Average cumulative dividends as a function of premium rate $c(\delta=0.05)$.

better precision than the 120 decimal digits we used for calculations. An interesting result of the algorithm is that it also gives all derivatives up to a given order, with respect to $u$ or in $v$, of $\operatorname{WF}(u, v)$. To give a concise illustration of section 4.1, figure 7 draws the evolution of average cumulative dividends that may be paid each time the process reaches the upper barrier without having reached the lower one. This simple, natural example is based on quantities computed in approximation or bounding algorithm. It is given here in a simplified environment, and introduction of other economical parameters, such as a discounting factor, would require further analysis.

\subsection{Comparison with other methods}

Sundt and Teugels (1995) proposed several methods to compute $\psi_{\delta}(u)$. Each one is based on the value of $\psi_{\delta}(0)$. If these methods are used to compute $S(u)=\mathrm{WF}(0, u)=\frac{1-\psi_{\delta}(0)}{1-\psi_{\delta}(u)}$, then the result obtained depends on the value of $\psi_{\delta}(0)$. Sundt and Teugels (1995) proposed for example a recursive algorithm that we rewrite with our notations:

$$
\begin{aligned}
& \varphi_{\delta}(h k)^{[-1]}=\gamma^{-}\left(c \varphi_{\delta}(0)+\sum_{j=1}^{k} \varphi_{\delta}(h(k-j))^{[-1]} f_{j}^{+}\right) \\
& \varphi_{\delta}(h k)^{[+1]}=\gamma^{+}\left(c \varphi_{\delta}(0)+\sum_{j=1}^{k-1} \varphi_{\delta}(h(k-j))^{[+1]} f_{j}^{-}\right) .
\end{aligned}
$$


with $\gamma^{-}=\frac{1}{c+\delta h k}, \gamma^{+}=\frac{1}{c+\delta h k-f_{1}^{+}}$, and, in the special case of integer-valued claim amounts, $f_{k}^{+}=\delta h+\lambda h \mathbb{P}[W \geq h k], f_{k}^{-}=f_{k+1}^{+}, h \leq 1 . f_{1}=(\lambda+\delta) h$. Note that the corresponding formulae for this quantities in Sundt and Teugels (1995) (top of page 12) have to be switched. Let us try to minimize

$$
\Delta_{\varphi_{k}^{h}}=\varphi_{\delta}(h k)^{[+1]}-\varphi_{\delta}(h k)^{[-1]}
$$

under the (very) optimistic hypothesis that for $j<k, \Delta_{\varphi_{k-j}^{h}}=0$. Note that

$$
\varphi_{\delta}(u) F(u)>\mathbb{E}\left[\varphi_{\delta}(u-W) \mathbb{1}_{W \leq u}\right]
$$

Hence, after some omitted computations, with $u=h k$,

$$
\Delta_{\varphi_{k}^{h}}>\frac{\left(\varphi_{\delta}(h k)-\varphi_{\delta}(0)\right)(\lambda(1-F(h k))+\delta)}{c+\delta h k} h,
$$

As an example, in the case $\delta=0$, we can get from table 3 values for the right member of (22). With same numerical parameters, the minoration of $\Delta_{\varphi_{k}^{h}}$ changes from values $10^{-3}$ to $10^{-5}$ when $h k \in[1,10]$. To get the same precision level $10^{-\eta}$ as in table 4 , would require an $h$ smaller than $10^{-\eta+5}$, and a much higher complexity in $1 / h^{2}$ than with our method. One must add to this problem the error possibly made in $\varphi_{\delta}(0)$, which was supposed to be avoided, and the propagation error due to $\Delta_{\varphi_{k-j}^{h}}, j \leq k$.

Other methods might not be more efficient, except in the case where $\varphi_{\delta}(0)$ is precisely bounded, for as well small or large values of $\delta$. Besides, the adjustment functions do not in general provide directly two-sided bounds for ruin probabilities. They are particularly adapted to the case of large initial reserve, which does not correspond to the assumption made here. This shows that the method consisting in taking the quotient of two non-ruin probabilities (computed with methods efficient for that problem) is not adapted to our framework, and that the algorithm proposed in section 3 is more convenient to this problem.

\section{References}

Brekelmans, R., De Waegenaere, A., 2001. Approximating the finite-time ruin probability under interest force. Insurance Math. Econom. 29 (2), 217-229.

De Vylder, F. E., 1999. Numerical finite-time ruin probabilities by the PicardLefèvre formula. Scand. Actuar. J. (2), 97-105.

Dickson, D. C. M., Gray, J. R., 1984a. Approximations to ruin probability in the presence of an upper absorbing barrier. Scand. Actuar. J. (2), 105-115.

Dickson, D. C. M., Gray, J. R., 1984b. Exact solutions for ruin probability in the presence of an absorbing upper barrier. Scand. Actuar. J. (3), 174-186.

Frostig, E., 2004. The expected time to ruin in a risk process with constant barrier via martingales. Working paper . 
Gerber, H., Shiu, E., 1998. On the time value of ruin. North American Actuarial Journal 2 (1), 48-78.

Gerber, H. U., 1979. An introduction to mathematical risk theory. Vol. 8 of S.S. Heubner Foundation Monograph Series. University of Pennsylvania Wharton School S.S. Huebner Foundation for Insurance Education, Philadelphia, Pa., with a foreword by James C. Hickman.

Konstantinides, D., Tang, Q., Tsitsiashvili, G., 2002. Estimates for the ruin probability in the classical risk model with constant interest force in the presence of heavy tails. Insurance Math. Econom. 31 (3), 447-460.

Picard, P., Lefèvre, C., 1997. The probability of ruin in finite time with discrete claim size distribution. Scand. Actuar. J. (1), 58-69.

Rullière, D., Loisel, S., 2004. Another look at the picard-lefèvre formula for finite-time ruin probabilities. Insurance Math. Econom. 35 (2), 187-203.

Segerdahl, C.-O., 1942. Über einige risikotheoretische Fragestellungen. Skand. Aktuarietidskr. 25, 43-83.

Segerdahl, C.-O., 1970. On some distributions in time connected with the collective theory of risk. Skand. Aktuarietidskr. , 167-192.

Sundt, B., Teugels, J. L., 1995. Ruin estimates under interest force. Insurance Math. Econom. 16 (1), 7-22.

Sundt, B., Teugels, J. L., 1997. The adjustment function in ruin estimates under interest force. Insurance Math. Econom. 19 (2), 85-94.

Wang, N., Politis, K., 2002. Some characteristics of a surplus process in the presence of an upper barrier. Insurance Math. Econom. 30 (2), 231-241.

STÉPHANE LOISEL

Ecole ISFA - Site de Gerland - 50 avenue Tony Garnier

F-69366 LYON CEDEX 07, FRANCE

Tel: +33437287438 Fax: +33437287632

E-mail: stephane.loisel@univ-lyon1.fr

DiDIER RULLIÈRE

Ecole ISFA - Site de Gerland - 50 avenue Tony Garnier F-69366 LYON CEDEX 07, FRANCE

Tel: +33437287438 Fax: +33437287632

E-mail: rulliere@univ-lyon1.fr 